\title{
APROVECHAMIENTOS DE ESCORRENTÍAS SUPERFICIALES EVENTUALES Y DE SUBALVEOS EN LA RAMBLA DE ORIA-ALBOX (ALMERÍA) ${ }^{1}$
}

\author{
Encarnación Gil Meseguer \\ Departamento de Geografía. Universidad de Murcia
}

\section{RESUMEN}

En los medios semiáridos, es frecuente que los cursos de agua superficiales sean del tipo rambla o río-rambla. En ellos se producen esporádicas escorrentías superficiales tras las lluvias, y también recargas de freáticos próximos a la superficie o subálveos que se rellenan de agua de lluvia al infiltrarse entre las capas de sedimentos acumulados en el lecho de la rambla. De cabecera a cola, para su aprovechamiento, sobresalen una sucesión de sistemas de presa y boquera, que derivarán esas aguas ocasionales de la escorrentía superficial por canales laterales a las márgenes de la rambla para los llamados riegos de boquera. La construcción de pozos horizontales cubiertos del tipo galería drenante o filtrante, entre los que predominan las cimbras, alumbran caudales con objeto de permitir la molienda al ser conducidos a molinos de cubo, y después utilizar las aguas en riegos más intensivos. En la rambla de Oria-Albox, afluente de la margen izquierda del Almanzora, hemos localizado varios de estos sistemas de aprovechamiento de agua, algunos de sus elementos han sido abandonados (molinos de cubo y boqueras) y otros se han rehabilitado (caños, abrevaderos, lavaderos, balsas). El objeto de este trabajo es explicarlos desde el enfoque de aprovechamiento integral del agua y la metodología de estudio regional.

Palabras clave: Medios semiáridos; rambla; boquera, qanat, cimbra; molino de cubo; regadío; Sureste de España.

1. Proyecto 17.587. «Eficiencia y sostenibilidad en el uso del agua en las zonas regables del Trasvase Negratín-Almanzora: Modernización de regadíos». Agradecimiento a los vecinos de Albox D. José Miras Carrasco (Presidente de la C.R. de la Fuente del Marqués), y de Oria D. Juan Jiménez (de la Fuente del Cocón), por la información facilitada. Y al profesor D. José M. ${ }^{\mathrm{a}}$ Gómez Espín por la cesión de las fotos. 


\section{ABSTRACT \\ Uses of eventual superficial torrents and of subriverbeds in the boulevard of Oria-Albox (Almería)}

In the semiarid areas, it is frequent that superficial water courses are boulevard or river - boulevard like; in which sporadic superficial torrents and also recharges on phreatics close to the surface; that is to say, subriverbeds that the rainwater refills by infiltrating between the layers of sediments accumulated in the bed of the boulevard, take place after the rains. From head to tail for its use they stand out a system sequence of prey and cold sore to derive these occasional waters from the superficial torrent to the margins of the boulevard for the called cold sore irrigations. And the construction of covered horizontal wells, such as draining or infiltrating gallery, in which cimbras that first light the water flow to allow grinding by being led to bucket mills, and later being applied to more intensive irrigations, are the prevailing ones. In the boulevard of Oria-Albox, tributary of the left margin of the Almanzora, we have located several of these water use systems, some of its elements have been left (mills of bucket and cold sores) and others have been rehabilitated (pipes, drinking troughs, laundry rooms, rafts). The object of this work is to explain them from the approach of integral use of the water and the methodology of regional study.

Keywords: Semiarid areas, boulevard sluice, qanat, cimbra, bucket hydraulic mill, irrigation, South-east of Spain.

\section{INTRODUCCIÓN. LOCALIZACIÓN. OBJETO Y MÉTODO}

La rambla de Oria-Albox, es afluente por su margen izquierda del río Almanzora, localizado en el territorio oriental de la provincia de Alicante (SE de España). La mayor parte de su cabecera se encuentra en la Sierra de las Estancias, en los glacis de acumulación que descienden de ella hacia el Sur y Este, a los que ha lacerado hasta la posición actual de los talweg de su red hidrográfica. A través del estrecho de la Boca de Oria se amplía su cuenca vertiente al captar buena parte de las escorrentías de los Llanos de Chirivel hasta los caseríos de Los Álamos, Los Quicos y la Yegua Alta, casi en el límite de Las Vertientes. Su afluente más importante es la rambla del Saliente, que converge, en la margen izquierda de la rambla principal, a la altura del Llano de Los Olleres, desde dónde toma la denominación de rambla de Albox hasta la confluencia con el curso medio del Almanzora, a la altura del Alto del Marchal, en las cercanías de la estación de Albox y el Cortijo de la Marquesa, en Las Casicas, entre las localidades de Cantoria (aguas arriba) y Arboleas (aguas abajo).

A mediados del siglo XIX, Pascual MADOZ en su Diccionario describía así las principales fuentes del término de Albox: «Albox dispone de algunas fuentes perennes: las más notables son la Fuente del Marqués, que riega la mayor parte 
de la vega hasta desembocar en el río Almanzora; la de Los Caños, con seis caños y abrevadero para las bestias; con la corriente de sus aguas se forma un pequeño álveo dónde se lavan las ropas de los vecinos; y otra fuente que brota a espalda del Santuario del Saliente, que aunque escasa se tiene por más exquisita y hasta como medicinal». Respecto a la rambla de Oria: «La rambla de Oria tiene su origen en el sitio llamado Las Vertientes, jurisdicción de Cúllar Baza, seis leguas distante de esta villa, y en el Campo de Cisnares, que lo es de Oria; recibe las aguas de tres ramblas denominadas de Oria, Medina, y Saliente, que se unen a media legua al Norte de la villa (de Albox), infiltrándose en las arenas de donde salen a la superficie por medio de zanjas y cimbras para los riegos y dar movimiento a los molinos harineros. La rambla va a desaguar, perdiendo su nombre, en el río Almanzora, a una legua de Albox, en el punto llamado Las Casicas». (Cerdán, D. 2014:163).

Salvo en la cabecera (tramo alto) que coincide con relieves como la Sierra de Las Estancias (vértice Pelado a 1.477 metros s.n.m.y Estancias a 1.467 metros s.n.m.), la Sierra de Oria (vértice Zurrio a 1.380 metros s.n.m.), La Sierra del Saliente (vértice Saliente a 1.501 metros s.n.m.), y el sector de los Llanos de Chirivel (entre los 1.000 y 1.100 metros s.n.m.); la mayor parte de la cuenca (tramos medio y bajo) es un territorio semiárido al coincidir escasas e irregulares precipitaciones con elevada insolación y temperaturas. Se suceden acusadas sequías (sobre todo en verano), con aguaceros de fuerte intensidad horaria que originan escorrentías superficiales y posibilitan la recarga de freáticos próximos en algunos lugares del lecho de la rambla.

Los turbiones aprovechables, no son sólo los de las grandes avenidas (que hacen a veces daños en las infraestructuras hidráulicas), también los que se generan tras «aguaduchos» cuya escorrentía superficial, en el cauce de la rambla, es fácil de derivar lateralmente mediante la asociación de presaboquera, conduciéndola por el margen de la rambla para mejorar secanos («riegos de alfait»), denominados riegos de boquera. Son presas de material suelto (arenas, gravas, cantos, restos vegetales amontonados), que forman un caballón de menos de un metro de altura y que se construye transversal al cauce pero sin ocuparlo totalmente para permitir que parte del caudal siga circulando aguas abajo.

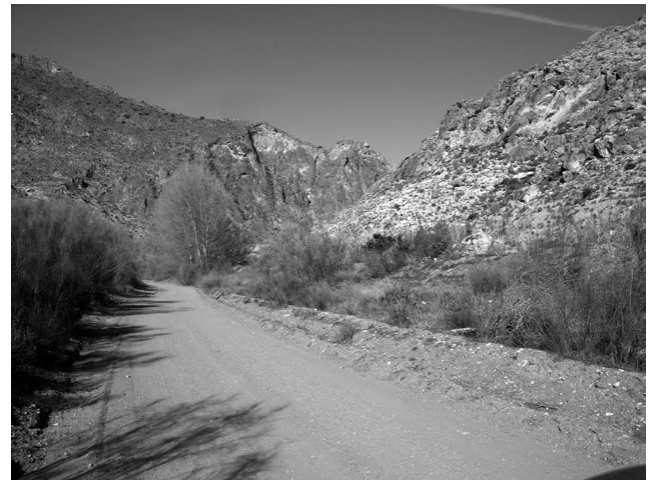

Figura 1. Rambla de Oria-Albox, aguas abajo de Los Estrechos. Fuente: Gómez Espín, J.M. ${ }^{a}$ $14 / 01 / 2015$. 
Aguaduchos, turbiones, crecidas y avenidas permiten la recarga de los freáticos formados en la acumulación de arrastres y posterior sedimentación en ciertos lugares del recorrido del curso. En ellos (álveos como pequeños vasos de embalse subterráneos), además de la circulación subsuperficial, si se saturan totalmente pueden manifestarse las aguas en la superficie del lecho unas horas e incluso unos días tras las lluvias. Las aguas subsuperficiales son alumbradas mediante la técnica del pozo horizontal (galerías del tipo tajeas o cimbras), usándose por lo general primero para abastecimiento de personas y ganados, usos domésticos e industriales, y finalmente para regadío.

Tras cada aguacero, el turbión tiene una determinada energía de arrastre de la corriente, cuando disminuye se produce «un deslastre» o acumulación de la carga, por lo que aumenta la cota del lecho. Así tras las precipitaciones y escorrentías se asiste a cambios en la distribución de los sedimentos del cauce y lecho. (Pulido, A. 1993: 132). Éstos, están la mayor parte del año secos, y no se observa circulación superficial de aguas. Situación que al coincidir con topografías suaves, pues se consideran lechos planos, facilitan su uso como vías de comunicación.

El objeto de este estudio es explicar los espacios de secano en los márgenes de la rambla regados de forma ocasional con las aguas de escorrentía superficial, derivadas en el lecho mediante sistemas en los que se asocia presa y boquera, para riegos eventuales de boquera (secanos asistidos por las aguas conducidas esporádicamente por la boquera). También las laderas subáridas de esta cuenca hidrográfica, que aprovechan las aguas pluviales caídas durante las precipitaciones al construir terrazas y abancalar esas vertientes. Se cierra cada terraza mediante un bordo interceptador de agua y suelo, «caballón», generalmente

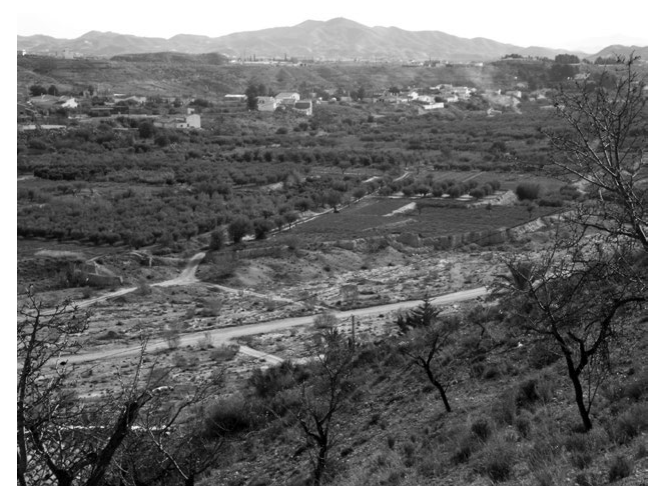

Figura 2. Espacio regado, mediante derivación de aguas de escorrentías superficiales eventuales y de alumbradas entandadas, en la margen izquierda de la rambla de Albox, (aguas abajo del acueducto de la conexión Negratía-Almanzora).

Fuente: Gómez Espín, J.M. ${ }^{a}$ 14/01/2015. con aliviaderos o sangradores para facilitar el paso del bancal superior al siguiente de menor altitud, y así sucesivamente. Son los riegos eventuales de ladera, en ocasiones asociados también a boqueras de vertiente. (Morales, A. 1968/69). Las aguas que después de aprovechamientos aún circulan superficialmente y se infiltran en los depósitos que rellenan el lecho de la rambla, constituyen álveos con circulación subsuperficial o subálvea, de donde son alumbradas mediante pozo horizontal (galería), y ordenadas en tandas para su aprovechamiento. (Llovet, S. 
1958). Con estas técnicas se mejoran secanos, se aseguran regadíos, y en general aumenta la productividad de estas tierras, en paisajes ordenados para la distribución de las aguas.

En cuanto a los usos del agua, se produce una sucesión de ellos. Primero el abastecimiento humano, sobre todo las aguas alumbradas mediante cimbras que suelen ser de gran calidad (filtradas entre arenas y gravas), también abrevaderos para el ganado, lavaderos

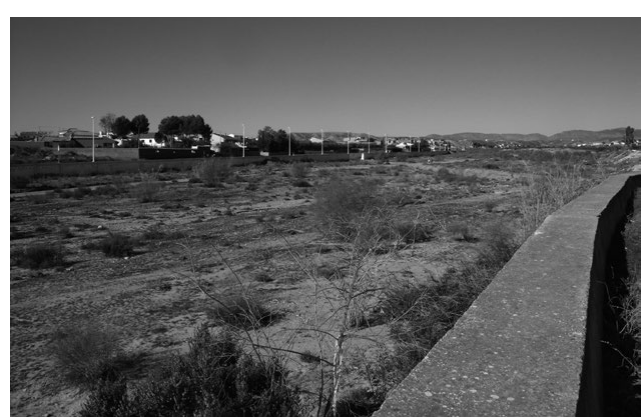

Figura 3. Encauzamiento de la Rambla de Albox, entre el núcleo del Pueblo y la entidad de $\mathrm{La}$ Loma construido tras la avenida del 19 de octubre de 1973. Fuente: Gil Meseguer, E y Gómez Espín, J.M. ${ }^{a} 04 / 01 / 2015$. relacionados con el uso doméstico, uso industrial en molinos de cubo y almazaras, y por último su uso en riegos eventuales y en permanentes entandados.

La ocupación del cauce para usos agrarios, urbanos y otros, genera graves daños en los aprovechamientos por las crecidas e inundaciones tras aguaceros de fuerte intensidad horaria (asociada en ocasiones a fenómenos de «gota fría», DANA, y con las altas temperaturas en las aguas del Mediterráneo Occidental sobre todo a finales del verano e inicios del otoño), En los últimos 130 años destacan avenidas como la del 14 de octubre de 1879 (Riada de Santa Teresa), el 11 de septiembre de 1891, la del 26 de junio de 1900, en el 19 de octubre de 1973, y la del 28 de septiembre de 2012. Los daños acostumbran a ser mayores por situaciones de ocupación indebida del cauce, como dice la sabiduría popular «la rambla sale con sus escrituras debajo del brazo». (Gil, E., et al. 2012).

El análisis, diagnóstico y prognosis de estos espacios y usos, nos permitirá recuperar algunos aprovechamientos, especialmente ciertos elementos de los sistemas de captación, distribución y acumulación del agua, y también conocer la gestión social de ella. Las salidas de campo, el estudio de las fuentes documentales y las entrevistas son la base metodológica de este estudio regional, con un enfoque de aprovechamiento integral del agua de una rambla (como la de Nogalte) (Gómez, J.M. a 2004).

APROVECHAMIENTOS DE TURBIONES EN RIEGOS DE BOQUERA DE LAS MARGENES de la Rambla de Oria-Albox. Abancalamientos de laderas SUbáridas PARA RIEGOS EVENTUALES, ASISTIDOS MEDIANTE CUENCAS DE CAPTACIÓN DE PLUVIALES Y BOQUERAS DE VERTIENTE

La topografía del valle de la rambla de Oria-Albox, en sus tramos medio y alto, es una sucesión de estrechos y de lugares de mayor amplitud (los llanos). 


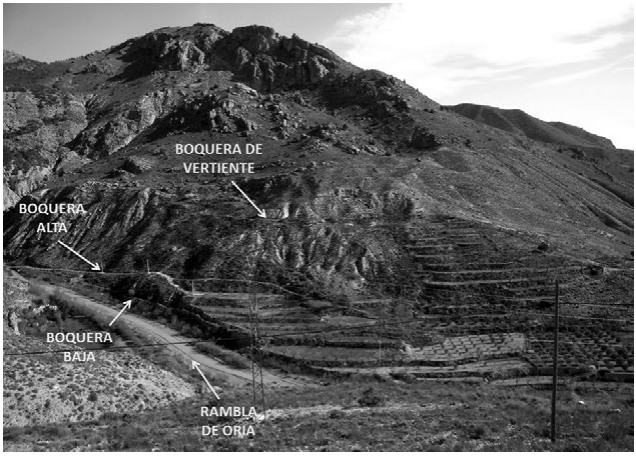

Figura 4. Paisajes de la margen izquierda de la rambla de Oria-Albox. En la parte superior, el riego eventual de laderas subáridas con bancales escalonados y boquera de vertiente. En la parte inferior dos sistemas de derivación de escorrentías superficiales eventuales del lecho fluvial (boqueras baja y alta).

Fuente: Gómez Espín, J.M.a 14/01/2015.

Una forma de «rosario», en la que apenas hay espacio en sus márgenes para establecer riegos eventuales de boquera con las parcelas alargadas en el sentido de la pendiente de la rambla. Desde el Llano de Los Olleres (540 metros s.n.m.) hasta la Boca de Oria (960 metros s.n.m.), se suceden estas «cuerdas» de regadíos a una y otra margen: Llano de las Ánimas, Llano del Espino, etc.,.y el diseminado poblacional: Los Simones, El Mojón, Los Charcones, Los Quiles, Los Jacintos, Los Gazques, Los Adrianes, etc. Las isohipsas de $600,700,800$ y hasta la de 900 metros s.n.m. enmarcan este tramo del valle. Las vertientes presentan acusadas pendientes, las masas de relieve parecen caer al lecho de la rambla, «se te vienen encima».

En la margen izquierda de la rambla de Oria, cercana a Los Timoteos, se observan estos espacios de «tablas» alargadas de secanos asistidos, «riegos de alfait», mediante las eventuales aguas de escorrentía superficial derivadas del cauce de la rambla por dos boqueras. La conducción más baja está excavada en la margen izquierda, con la toma y primeros metros de canal con un muro de obra en la cara más próxima a la rambla para darle más consistencia y protegerla de las avenidas. En los primeros cincuenta metros cuenta con una balsa cuadrada para acumulación de una fracción del agua derivada, y cien metros más abajo se inicia la distribución del líquido elemento en bancales alargados pero con gran anchura, que permite varias hileras de árboles e incluso la mecanización de labores como arado y fumigación con tractores. Cada bancal dispone de caballón de casi un metro de altura que le rodea y permite inundar la caja, en algunos de estos bordos interceptadores se han situado, a amplias distancias, arbolado (almendros). También, cuentan con aliviaderos, «sangradores», para que por gravedad y según la pendiente, pase el agua turbia del bancal primero o superior (cuando esté suficientemente regado), al siguiente y así sucesivamente; en el último la «cola» vierte a la rambla.

La boquera de arriba, que deriva el turbión a cota más alta, está excavada en el piedemonte, y en los trescientos primeros metros es un canal a cielo abierto que además de conducir las aguas derivadas del lecho de la rambla, intercepta y recoge todas las escorrentías, «escurrimbles», de esa margen que laceran la 
parte inferior de la vertiente que está sin transformar para cultivo, por lo que también se convierte en boquera de vertiente. Para derivar el agua de la rambla, la boquera parece prolongarse en su cara más externa, como una mota o presa simple (de tierra fagina) sin ocupar totalmente el cauce. Los bancales presentan formas más alargadas, están escalonados y mantienen el concepto de cajón inundable con caballón perimetral y aliviadero o sangrador asociado. La dedicación de estos riegos de boquera fue de

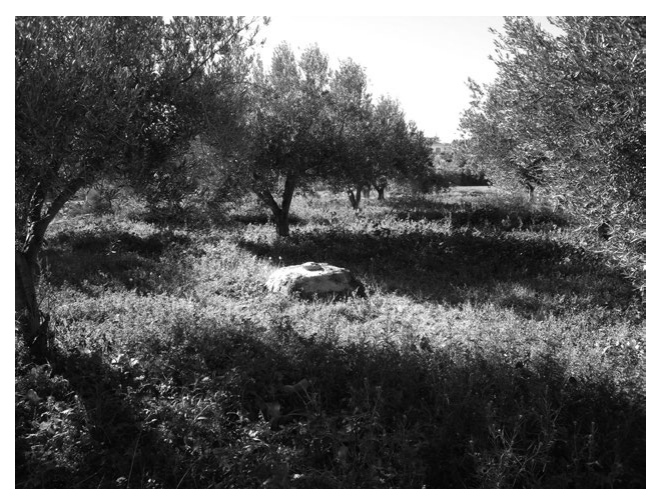

Figura 5. Tercera lumbrera del qanat de la Fuente del Marqués, en el paraje Locaíba, en la margen izquierda de la rambla de Albox.

Fuente Gil, E.; Gómez, J.M. ${ }^{\text {a }}$ 04/01/2015. cereal, con arbolado (almendros) en los bordes, junto al margen En la actualidad los regadíos de este tipo, que no están abandonados, se dedican sobre todo al cultivo de olivar. En los espacios abandonados se observa una recuperación de la vegetación natural mediterránea, ahora bien los «costones» de tierra de las motas se están destruyendo por la acción de roedores y de las aguas de arroyada que han formado los coloquialmente llamados «trenques».

En la ladera, hacia la mitad se ha abancalado adaptándose cada uno de los bancales (estrechos y arqueados) como a la forma de un cono de deyección (cortos y estrechos los superiores y más alargados pero estrechos los inferiores, apenas para un hilera de árboles). Cuentan con caballón, pero sólo en el frente del lado de la pendiente mientras que los extremos permiten el paso de las aguas al siguiente bancal o terraza. Además de la precipitación que les cae, reciben para riego eventual las aguas de lluvia caídas en la cuenca receptora en que se convierte la ladera no transformada por encima del abancalamiento, a través de una boquera de vertiente. (Hernández, M; Morales, A. 2013). Los ribazos o costones que separan un bancal de otro, son de grandes dimensiones por las fuertes pendiente de la ladera. $\mathrm{Al}$ abandonarse se ha desorganizado el riego, y se produce un proceso de regulación de la vertiente con destrucción de buena parte de los bancales. (Lasanta, T., et al. 2013).

 ALUMBRADAS SUS AGUAS POR GALERÍAS}

Los lechos de las ramblas suelen ser de permeabilidad elevada, aunque variable en el espacio. Este rasgo favorece la infiltración en algunos lugares y tramos 


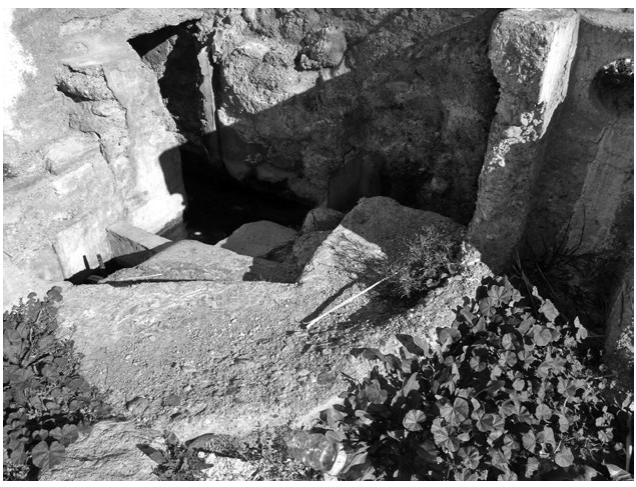

Figura 6. Bocamina y partidor del qanat Fuente del Marqués, paraje Aljara, en la margen izquierda de la rambla de Albox.

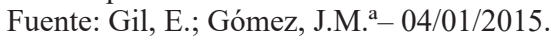

que cuentan con rellenos del lecho de gran permeabilidad vertical y horizontal. Tras las lluvias, junto a la consiguiente escorrentía superficial, las aguas se infiltran en estos depósitos de sedimentos hasta su saturación. Se constituyen como pequeños embalses subterráneos de los que el agua será extraída por pozos verticales y artefacto elevador asociado (garrucha, polea, torno, noria de tiro, etc.,) y sobre todo alumbrada por pozos horizontales cubiertos (galerías) del tipo cimbra o qanat.

En la margen izquierda de la rambla de Albox y frente al núcleo de población, en el pago Aljara, la cimbra de la Fuente de Los Caños, con abrevadero y lavadero, abasteció durante un tiempo a la población de Albox y de La Loma. Las avenidas de 1775 y de 1778 destruyeron los elementos asociados y la acequia común que conducía las aguas al riego del pago de la Aljara. En febrero de 1791 el Marqués de los Vélez concedió un préstamo que permitió sufragar el alto coste de la reparación. (Cerdán, D. 2014:146). Las avenidas de 10 de octubre de 1966 y sobre todo la del 19 de octubre de 1973, destruyeron esas infraestructuras hidráulicas e inundaron el pago de la Aljara y otros. (En Albox se recogieron 40 1/ $\mathrm{m}^{2}$ el día 17, 134 1/ $\mathrm{m}^{2}$ el día 18, y $113 \mathrm{l} / \mathrm{m}^{2}$ el día 19 , es decir $287 \mathrm{l} / \mathrm{m}^{2}$ en total. En otros lugares, como Zurgena, casi se triplicaron esos volúmenes, pues sólo los dos últimos días se recogieron $7301 / \mathrm{m}^{2}$ ). También se vieron afectados los molinos hidráulicos de cubo, situados en el trazado de la red de distribución de agua de la cimbra de la Fuente de Los Caños, como el molino de «La Chuza» y el de Ángel Saéz Serrano.

En la margen izquierda, aguas arriba en el pago de Locaiba, se encuentra el pozo madre, en el que brotan las aguas entre las arenas a 28 metros de profundidad, del qanat de la Fuente del Marqués, y tres de las lumbreras (pozos verticales a la galería). De ahí atraviesa a la otra margen como «presa subálvea» y riega el pago Ruiz, para volver de nuevo a la margen izquierda, donde todavía se observa la última lumbrera, la bocamina y las conducciones para riego en los pagos de Aljara, San Francisco, El Dirá, etc. En la primera quincena de enero de 2015 los aforos variaban de 7 a 10 litros por segundo. Las aguas se repartían en tanda de 31 días, para el riego de una huerta periurbana de cultivos de vuelo de olivar y cítricos, en ocasiones asociados a cultivos de suelo (tubérculos y hortalizas). Hasta el último tercio del siglo xx a lo largo del recorrido de las 
aguas alumbradas, en su trayecto para riego del espacio cultivado que a pesar de haber reducido su extensión por la expansión urbana aún existe, éstas movían las piedras de cuatro molinos hidráulicos de cubo: el de Antonio «el Señorito», el de Balibrea, el de Carlos, y el de «Calicanto».

En esta rambla de Albox, en la margen derecha, aguas arriba de la población que le da nombre, alumbraba aguas el minado de la Fuente del Pleito, con balsa para acumular las aguas y aprovechamiento en

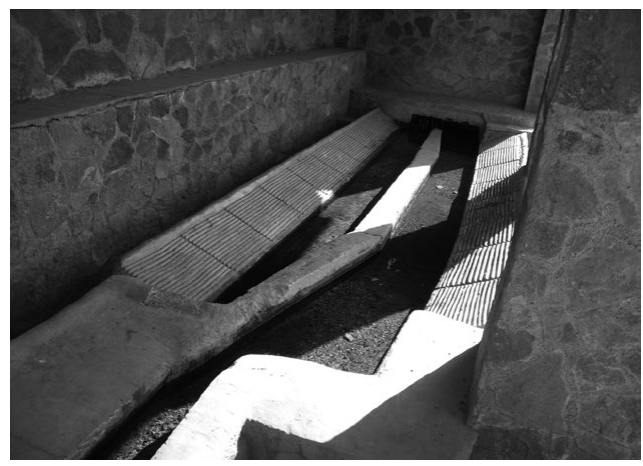

Figura 7. Lavadero rehabilitado, a continuación de la bocamina de la cimbra de la Fuente de Las Mercedes, en la margen izquierda de la rambla de Oria. Fuente: Gómez Espín, J.M. ${ }^{a}$ 14/01/2015. la industria molinera como el Molino de Las Piedras. En esta misma margen, con las aguas de la Fuente de Los Olleres se movían los molinos hidráulicos de Cipriano y de los «Tadeos». También se alumbran más aguas mediante la cimbra de la Fuente de La Alcaina, cuyo riego con esos caudales se extendía por la Chinica de la Alcaina, en tanda desde 1757, y las sobrantes a los pagos de Locaiba y San Roque, en tanda desde 1772. (Cerdá, D. 2014: 138). En la Terrera de la Alcaina se movían las piedras de molinos hidráulicos de cubo como los de Matías García y el del «Violín». En la misma margen derecha, aguas más arriba, figuran las alumbradas por la cimbra de la Fuente del Mojón y Llano del Espino, que movían las piedras de los molinos del Rincón (de José Miras), del Mellizo, el de José Olula, el del «Pintao» y el de «Frasquito el Molinero».

En la margen izquierda de la rambla de Oria, la cimbra de la Fuente de Las Mercedes, se nos presenta con la bocamina, caños, abrevadero y lavadero rehabilitados y mejorado el inicio de las conducciones a la zona de riego; en ese trayecto funcionaron varios molinos hidráulicos de cubo como el de Paco «el Molinero», el de Paco Mira, y el de José «Cabila». Apenas tres kilómetros aguas arriba en esa margen izquierda también alumbran aguas de la rambla la cimbra de la Fuente del Ángel».

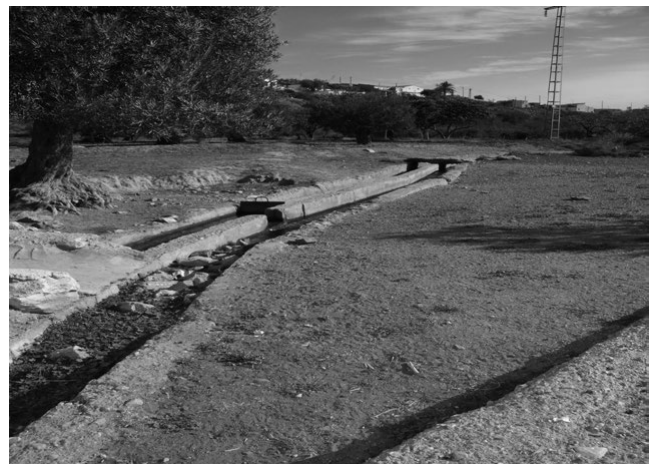

Figura 8. Conducciones de distribución de las aguas alumbradas por la cimbra de la Fuente de Las Mercedes. Fuente: Gómez Espín, J.M. ${ }^{\mathrm{a}}$ $14 / 01 / 2015$. 


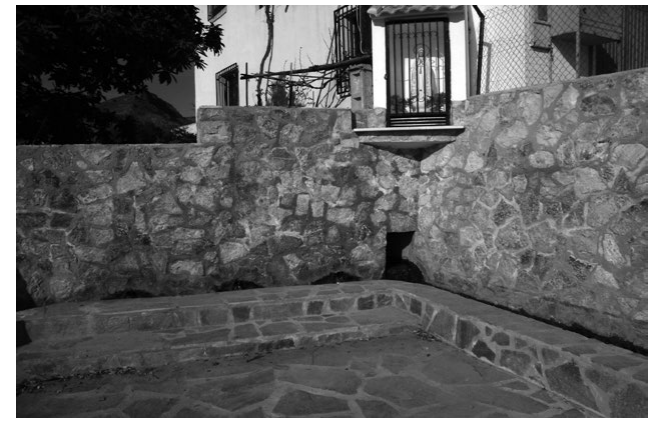

Figura 9: Bocamina y caños de la cimbra de la Fuente del Cocón. Fuente: Gómez Espín, J.M. ${ }^{\mathrm{a}}$ 14/01/2015.

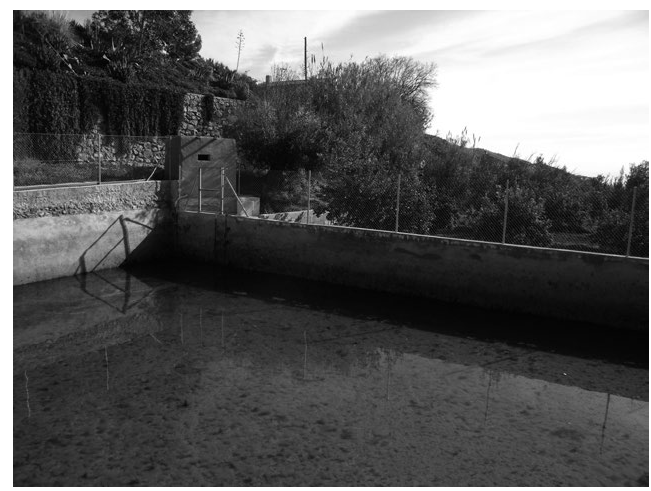

Figura 10. Balsa que acumula parte del agua alumbrada por la cimbra del Cocón.

Fuente: Gómez Espín, J.M.a 14/01/2015.

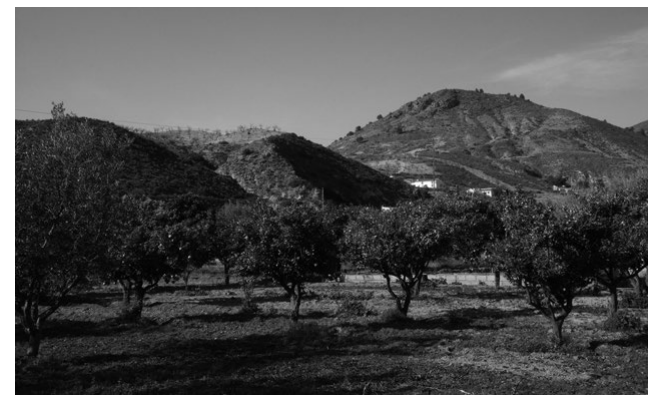

Figura 11. Espacio de policultivo en la margen izquierda de la rambla de Oria, regable con las aguas alumbradas por la galería de la Fuente del Cocón. Fuente: Gómez Espín, J.M. a 14/01/2015.
Siguiendo en esa margen izquierda, en término de Oria, sobresale la cimbra de la Fuente del Cocón. Están rehabilitados la bocamina, caños, abrevadero, lavadero y balsa (de forma rectangular). El espacio regable está dedicado a un policultivo arbóreo de frutales y cítricos, en ocasiones asociados a cultivos de suelo (hortícolas). En el recorrido de distribución de las aguas se cuenta con industria molinar como el Molino del Peñon de Abajo (de José García Galera).

Aguas abajo de la Boca de Oria sobresalen los restos de un conjunto molinar, en ambas márgenes de la rambla, como los de Andrés Campoy, el del «Arriero», el de José Quiles, el de Paco Lázaro «Chavirras», el de Fernando Aís «Fernandón», el de Los Quiles, el del «Chispero», de José Mata, el de Fernando Miras, etc.

En la rambla del Saliente también se cuenta con fuentes y molinos hidráulicos asociados a las aguas alumbradas. Entre las fuentes sobresalian: la de Santa Rosa (caños, abrevadero, lavadero y balsa), la del Carmen, la de San Rafael, etc. Los molinos hidráulicos de cubo están escalonados en función de la pendientede de este curso afluente. A mediados del siglo Xx figuraban, de arriba abajo, el del Tio Bautista, el de Antonia «La Molinera», el de Juan Roa», el de Ramón Rosendo, el de Ginés Mirón, el de Roque Jiménez, el de Antonio Martínez. A partir de 
la convergencia de la rambla del Saliente en la margen izquierda de la de Oria, el curso se denomina rambla de Albox hasta la desembocadura en el Almanzora. Cerca de ésta, y en la rambla de Albox, también habia molinos como los de Juan Mellado, el del «Pulpito» y el de Consuelo Águila.

\section{CONCLUSIONES Y PROPUESTAS}

En la cuenca de drenaje de la rambla de Oria-Albox, y su afluente

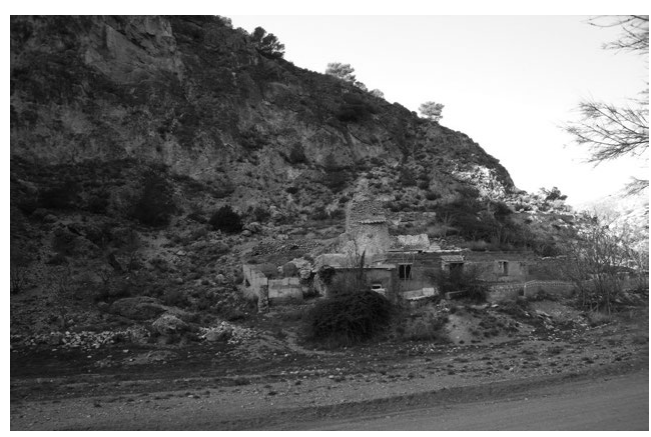

Figura 12. Molino hidráulico de cubo en la margen derecha de la rambla de Oria, aguas abajo de la Boca de Oria.Fuente: Gómez Espín, J.M. ${ }^{a}$ 14/01/2015. la rambla del Saliente, pueden observarse aprovechamientos de pluviales y de escorrentía reducida en laderas subáridas, con aterrazamientos y sangradores, e incluso boqueras de vertiente. Sistemas de aprovechamiento de aguas eventuales de escorrentía superficial concentradas en las ramblas y, captadas por derivación desde el cauce a partir de presa y boquera, para riegos de boquera. Alumbramientos de freáticos próximos (subálveos) en sedimentos del cauce y lecho de la rambla, mediante la técnica del pozo horizontal (galerías) y elementos anexos como bocamina, caños, abrevadero, lavadero, balsas, conducciones, etc., para abastecimiento de personas y ganados, usos domésticos e industriales, y para riego. Esta sucesión de hitos hidraúlicos hablan de un aprovechamiento integral de las escasas e irregulares aguas de escorrentía de este espacio. Es el resultado de una ordenación del territorio que, además de permitir el establecimiento de un poblamiento, creó un rico patrimonio hidraúlico en parte abandonado hoy.

Algunos de estos elementos se han rehabilitado como las cimbras de la Fuente de Las Mercedes y de la Fuente del Cocón. Otros necesitan de limpieza y mejoras en el sistema de captación y alumbramiento como el qanat de la Fuente del Marqués. El mantenimiento puede ser a partir de ayudas al desarrollo rural para aquellos agricultores que no abandonen sistemas de riegos de boquera y riegos en laderas subáridas. Se podrían rehabilitar algunos de los complejos molinares hidráulicos de cubo, por su adaptación a las condiciones de los medios semiáridos, y preparar unas sendas del agua, unas rutas de interés basadas en estos paisajes y elementos patrimoniales, para no olvidar el papel que todas las técnicas han tenido y tienen en alumbrar y disponer de recursos propios de agua. 


\section{FUENTES Y BIBLIOGRAFÍA}

Cerdán Galera, D. (2014): Hitos y efemérides de la Historia de Albox, siglo XII-1975. Ediciones Marcianas. Madrid. 541 pp.

Fernández Bolea, E. (2006): Agua y vida en Cuevas de Almanzora. Una historia de luchas y anhelos (siglos XVI-XXI). Arráez Editores, S.L., Mojacar (Almería). 334 pp.

FERnÁNDEZ BoleA, E. (2007):» El pantano de Nijar: antecedentes y primeros tiempos de una empresa frustrada. Revista Axarquía, n. ${ }^{\circ} 12$. Ayuntamiento de Níjar. Pp.145-160.

Ferre Bueno, E. (1979): El Valle del Almanzora. Estudio Geográfico. Excma. Diputación Provincial. Caja Rural Provincial. Monte de Piedad y Caja de Ahorros de Almería. Almería. 494 pp.

Gil Meseguer, E. (2014):«Aprovechamiento y gestión del agua en medio semiáridos». PhICARIa II Encuentros Internacionales del Mediterráneo. Uso y gestión de recursos naturales en medios semiáridos de ámbito mediterráneo. Univ. Popular de Mazarrón. Pp.39-52.

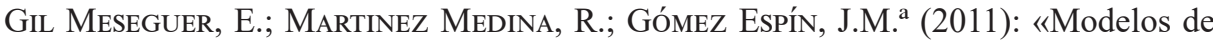
uso sostenible del agua: las galerías asociadas a presa subálvea». Scripta Nova. Revista Electrónica de Geografía y Ciencias Sociales. Volumen XV, $n .{ }^{\circ} 374.10 \mathrm{de}$ septiembre de 2011. Barcelona. Pp.1-16.

Gil Meseguer, E.; Gómez Espín, J. M.a (2012): «La investigación en España sobre los sistemas de captación y conducción de pozo horizontal (galería), asociada o no a presa subálvea». Patrimonio hidráulico y cultura del agua en el Mediterráneo. Fundación Séneca. AECID. Campus de Excelencia Internacional «Mare Nostrum». Murcia. Pp.189-202.

Gil Meseguer, E.: Gómez Espín, J.M.a (2014):» Las aguas de fuentes en Huércal-Overa (Almería). El modelo de la Sociedad Colectiva de Aguas titulada $<$ La Asunción $>$ ». Libro jubilar en homenaje al Profesor Antonio Gil Olcina. Universidad de Alicante. Pp.215-230

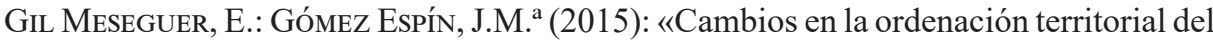
Bajo Almanzora auspiciados por los trasvase Tajo-Segura y Negratín-Almanzora». De la Riva, J.; Ibarra, P.; Montorio, R.; Rodríguez, M. (Eds.). Análisis espacial y representación geográfica: innovación y aplicación. Zaragoza. Pp.139-147.

Gil Meseguer, E.; Pérez Morales, A.; Gómez Espín, J.M. (2012): « Precipitaciones y avenidas del 28 de septiembre de 2012 en el cuadrante suroccidental de la Cuenca del Segura (municipios de Lorca, Puerto Lumbreras y Pulpí)». Papeles de Geografía, n. ${ }^{\circ}$ 55-56. Universidad de Murcia. Pp.75-94.

Gil Meseguer, E.; García Martinez, P.J.; Gómez Espín, J.M.a; Almela Pérez, R. (2014): El dinamismo del regadio de Pulpí. Comunidad de Regantes de Pulpí. Murcia. $222 \mathrm{pp}$.

Gil Meseguer, E.; López Fernández, J.A.; Gómez Espín, J: M. ${ }^{\text {a }}$ (2015): «Regadíos de turbias y secanos asistidos en el Sureste de España. El sistema del Azud de Guadalupe o presa de derivación de avenidas de la Rambla del Zoco». Revista Murciana de Antropología, ${ }^{\circ}{ }^{\circ} 22$. (Número monográfico de Cultura del Agua en Murcia y Sureste Español). Universidad de Murcia. Pp. 161-176. 
Gil OlcinA, A. (1993): La propiedad de las aguas perennes en el Sureste ibérico. Servicio de Publicaciones. Universidad de Alicante. $191 \mathrm{pp}$.

Gómez Espín, J. M. ${ }^{\mathrm{a}}$ (2004): Aprovechamiento integral del agua de la Rambla de Nogalte (Puerto lumbreras-Murcia). CAM Proyecto Agua. Ayuntamiento de Puerto Lumbreras. Universidad de Murcia. Murcia. 190 pp.

Hermosilla Plá, J. et al. (2004): «Las galerías drenantes de la provincia de Almería. Análisis, y clasificación tipológica». Cuadernos de Geografía, $n{ }^{\circ}{ }^{\circ} 76$. Universidad de Valencia. Pp.125-154.

Hermosilla Plá, J. (Director) (2010): Los nuevos regadios históricos españoles. Paisajes culturales, paisajes sostenibles. Ministerio de Medio Ambiente y Medio Rural y Marino. Madrid. 608 pp.

Hernández Hernández, M.; Morales Gil, A. (2013): «Los aprovechamientos tradicionales de las aguas turbias en los piedemontes del Sureste de la Península Ibérica». Boletín de la Asociación de Geógrafos Españoles, n. ${ }^{\circ}$ 63. Murcia. Pp.105-123.

JunTA DE ANDAlucía. (2010): Manantiales y fuentes de Andalucía. Hacia una estrategia de Conservación. Universidad de Granada. Junta de Andalucía. (http:/www. conocetusfuentes.com).

Lasanta, T.: ArnaÉz, J., Ruiz, P., Lans-Renault, N. (2013): «Los bancales en las montañas españolas: un paisaje abandonado y un recurso potencial». Boletín de la Asociación de Geógrafos Españoles), $n .^{\circ}$ 63. Murcia. Pp. 321-322

Llobet ReVERTER, S. (1958): «Utilización del suelo y economía del agua en la región semiárida de de Huércal-Overa (Almería)». Revista Estudios Geográficos, n. $^{\circ} 70$. Febrero 1958. Madrid. Pp. 5-21.

Madoz, P. (1845/50): Diccionario Geográfico-Estadístico-Histórico de España y sus Posesiones de Ultramar. Varios Tomos.

Morales Gil, A. (1968-1969): «El riego con aguas de avenidas en las laderas subáridas». Papeles de Geografía, n. ${ }^{\circ}$ 1. Universidad de Murcia. Pp.167-183.

Morales, A.; Box, M.; Marco, J.A. (1989): «El aprovechamiento de las aguas de avenida: derecho consuetudinario y disposiciones legales». Avenidas fluviales $e$ inundaciones en la Cuenca del Mediterráneo. Instituto Universitario de Geografía. Universidad de Alicante. CAM. Murcia. Pp.553-563.

Morales, A.; Box, M.; Marco, J.A. (1991): «El medio físico y la presa del Román (Jumilla): un emplazamiento favorable». Investigaciones Geográficas, n. ${ }^{\circ} 9$. Universidad de Alicante. Murcia. Pp.69-80.

Morote Seguido, A.F. (2013): «El aprovechamiento de turbias en San Vicente de Raspeig (Alicante) como ejemplo de sistema de riego tradicional y sostenible». Investigaciones Geográficas, $n .^{\circ}$ 59. Universidad de Alicante. Pp.147-169.

Pulido Bosch, A. (1993): «Las ramblas mediterráneas: condicionantes geomorfológicos e hidrológicos». Regeneración de la cubierta vegetal. Actas Aula de ecología. Pp. 131-140. 